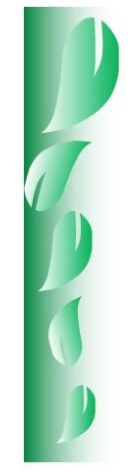

DOI: https://doi.org/10.29298/rmcf.v12iEspecial-1.1076

Artículo de revisión

\title{
Aportaciones del INIFAP en materia de plagas y enfermedades forestales
}

\section{INIFAP contributions on forest pests and diseases}

\author{
Guillermo Sánchez Martínez ${ }^{1 *}$ y Francisco Reséndiz Martínez²
}

\begin{abstract}
This paper aims to give information on the main contributions that the Instituto Nacional de Investigaciones Forestales, Agrícolas y Pecuarias (INIFAP) has made on forest pests and diseases during its first 35 years of existence. This study is based on a literature review that comprises a time since the foundation of the Instituto Nacional de Investigaciones Forestales, that precedes INIFAP, to present days. The contribution of both institutes, with knowledge on the taxonomic, biological, and control of conifer bark beetles (Dendroctonus spp. and Ips spp.), conifer sawflies (Zadiprion spp., Neodiprion spp. and Monoctenus spp.) as well as other tree defoliators of the Lepidoptera order and some exotic pests is outstanding. Regarding forest diseases, there are notable studies on the diagnosis of causal agents of rots and rusts, mainly fungi (e. g. Fusarium spp., Rhizoctonia spp., Cronartium, Colletotrichum spp.), and the diagnosis, impact, and control of parasitic plants in natural forests, urban trees and tree plantations. In the last years, the INIFAP contribution of knowledge that supports the NOM-019-SEMARNAT 2017 Official Mexican Norm, allusive to the prevention and control of bark beetles, and on the state-of-the-art on conifer sawflies stands out. This effort has been mainly oriented to the diagnosis and control of forest pests and diseases; however, INIFAP researchers face the challenge of generating information and technology that allows to prevent and mitigate the effects of climate change on forest health.
\end{abstract}

Key words: Forest diseases, pathogenic fungi, foliar insect, bark beetles, forest pests, forest health.

\section{Resumen}

El objetivo de este trabajo es proveer información sobre las principales aportaciones que ha hecho el Instituto Nacional de Investigaciones Forestales, Agrícolas y Pecuarias (INIFAP) en materia de plagas y enfermedades forestales, durante sus primeros 35 años de existencia. El estudio se basa en una revisión bibliográfica que comprende el periodo desde la fundación del Instituto Nacional de Investigaciones Forestales, antecesor del INIFAP, hasta el presente. Destaca la aportación de ambos institutos sobre el conocimiento taxonómico, biológico y de control de insectos descortezadores de coníferas (Dendroctonus spp. e Ips spp.), moscas sierra de las coníferas (Zadiprion spp., Neodiprion spp. y Monoctenus spp.), así como de otros defoliadores del orden Lepidoptera y algunas plagas exóticas. En cuanto a enfermedades forestales, son notables los estudios de diagnóstico de los agentes causales de pudriciones y royas, principalmente hongos (Fusarium spp., Rhizoctonia spp., Cronartium spp, Colletotrichum spp.) y el diagnóstico, impacto y control de plantas parásitas, tanto en bosques naturales como en arbolado urbano y plantaciones. En los últimos años, sobresale la contribución del INIFAP con conocimientos que fundamentan la Norma Oficial Mexicana NOM-019-SEMARNAT 2017, alusiva a la prevención y control de insectos descortezadores; y el estado del arte sobre las moscas sierra de las coníferas. Este esfuerzo se ha dirigido, fundalmente, al diagnóstico y control de plagas y enfermedades forestales; sin embargo, los investigadores del INIFAP enfrentan el reto de generar información y tecnología que permita prevenir y mitigar los efectos del cambio climático en la salud de los bosques.

Palabras clave: Enfermedades forestales, hongos patógenos, insectos defoliadores, insectos descortezadores, plagas forestales, salud forestal.

Fecha de recepción/Reception date: 31 de enero de 2021

Fecha de aceptación/Acceptance date: 13 de mayo de 2021

${ }^{1}$ Instituto Nacional de Investigaciones Forestales, Agrícolas y Pecuarias. Campo Experimental Pabellón. México. 2Instituto Nacional de Investigaciones Forestales, Agrícolas y Pecuarias. Centro Nacional de Investigación Disciplinaria en Conservación y Mejoramiento de Ecosistemas Forestales. México. 


\section{Introducción}

La sanidad forestal atiende la presencia de plagas y enfermedades en los bosques (CCAD, 2017). Según la FAO (2018), 70 \% del total de la superficie forestal mundial presenta daños por estas. En el ámbito de los ecosistemas naturales, la asignación del término plaga tiene una connotación antropocéntrica, pues muchos insectos y organismos así designados mantienen la dinámica de las comunidades vegetales y los procesos ecológicos mayores; sin los cuales, los bosques simplemente se estancarían o tendrían una pobre biodiversidad (Schowalter et al., 1997; Schowalter, 2012). En otros escenarios, tales como las plantaciones forestales comerciales o los bosques urbanos, la función ecológica de insectos y patógenos es secundaria, ya que se da prioridad a evitar daños económicos. Bajo esos diferentes contextos, es imperativo para las instituciones científicas y académicas realizar estudios pertinentes con el fin de desarrollar estrategias de manejo.

El objetivo del presente estudio consistió en proporcionar información sobre las principales aportaciones del Instituto Nacional de Investigaciones Forestales, Agrícolas y Pecuarias en materia de sanidad forestal, durante sus primeros 35 años, tanto en bosques naturales, plantaciones como en el arbolado urbano, a partir de los trabajos realizados en el Instituto Nacional de Investigaciones Forestales (INIF) que lo antecedió hasta el 2020, año en el que se conmemoraron los 35 años del INIFAP.

\section{INIF, un instituto antecesor}

Desde su fundación en 1958, el Instituto Nacional de Investigaciones Forestales (INIF) privilegió la investigación sobre plagas y enfermedades, por lo que se crearon los laboratorios de entomología y fitopatología forestal en la Ciudad de México (INIF, 1982), y se asignó personal investigador en el área de protección forestal en algunos Centros de Investigación Regional del país.

Desde 1959 y hasta 1985, la investigación en Sanidad Forestal del INIF se enfocó, principalmente, en estudios de identificación, biología y combate directo de plagas y 
enfermedades en bosques templados del centro y sur del país; aunque también, se abordaron aspectos de plagas y enfermedades en bosques urbanos de la Ciudad de México. Destacan en este periodo las aportaciones de Justina Perusquía Ortiz, quien describió todas las especies de Dendroctonus spp. conocidas para México, su distribución geográfica, así como sus enemigos naturales (Perusquía, 1978; 1982). Dicho género comprende las especies plaga de mayor importancia ecológica y económica para los bosques templados del Hemisferio Norte.

El conocimiento generado por Federico Islas Salas sobre la biología y el combate de los insectos descortezadores de pino Dendroctonus adjunctus Blandford, Dendroctonus mexicanus Hopkins y Dendroctonus frontalis Zimmerman (Coleoptera: Curculionidae: Scolytinae), particularmente en Ciudad de México, Estado de México, Puebla, Guerrero y Jalisco (Islas, 1968; 1980a) está considerado un esfuerzo de enorme trascendencia nacional.

Islas (1980b) validó la efectividad de los métodos físico-mecánicos de control de dichos insectos, consistentes en el derribo y descortezado de árboles infestados, y el "derribo y abandono", este último es un método en el cual el derribo temprano de los árboles infestados, sin remover la corteza, estimula la acción de los enemigos naturales y competidores. La efectividad de práctica se corroboró en el Parque Nacional Nevado de Colima, Jalisco, donde se observó el abatimiento de la población de $D$. adjunctus por la acción de depredadores naturales, competencia con especies secundarias y desecación (Villa, 1985; 1993).

Otros métodos de control explorados en investigaciones del INIF versaron sobre el uso de semioquímicos atrayentes de insectos descortezadores (frontalina, brevicomina y transverbenol) para la creación de árboles trampa, así como la validación y adaptación de técnicas antiguas de inyección de insecticidas, fungicidas, bactericidas y entomopatógenos originadas en Europa durante los siglos XVI y XVII (Vité et al., 1974; Islas, 1980a). Aunque, también se experimentó con la aplicación de algunos insecticidas sobre el fuste de los árboles, el INIF 
desde un principio recomendó no usarlos en bosques naturales por resultar tóxicos a otros organismos (Islas, 1980b).

En el INIF se impulsó el estudio del grupo de insectos defoliadores conocidos como "moscas sierra de las coníferas" (Hymenoptera: Diprionidae), cuyos primeros registros en México datan de 1912 y 1930 (Mayo, 1976). Las defoliaciones de pino causadas por estos insectos en los años 70 en Michoacán, y principios de los años 80 en Chihuahua resultaron en la determinación de tres especies de mosca sierra: Zadiprion vallicola Rohwer, Neodiprion sp. Circa gillettei Rohwer y Neodiprion fulviceps Cresson; así como en el conocimiento detallado de su biología y control (Cisneros, 1970; Mayo, 1976; Castro, 1981; Islas y Muñiz, 1985). Estudios posteriores actualizaron el estatus de dichas especies, y las reclasificaron como Zadiprion falsus Smith, Neodiprion omosus Smith y Neodiprion autumnalis Smith, respectivamente (Smith y Wagner, 1986; Smith, 1988).

En el contexto citadino, la contribución de Gutiérrez y Muñiz, (1984) en la identificación de las principales especies forestales y de las plagas de insectos que afectan al Bosque de Chapultepec en la Ciudad de México fue una de las primeras aportaciones en el campo de las áreas verdes urbanas.

En cuanto al estudio de las enfermedades forestales, es digno de mención el trabajo realizado por Rodolfo Salinas Quinard, quien dirigió el Laboratorio de Patología Forestal y fue un dedicado investigador del área de insectos y enfermedades forestales del INIF. Entre sus principales logros científicos destacan el desarrollo de métodos de laboratorio para evaluar la resistencia de la madera a las pudriciones (Gómez et al., 1969; Pérez y Salinas, 1977), y el de técnicas para detectar hongos asociados con Dendroctonus spp., causantes del manchado azul de la madera (Salinas y Macías, 1979). Otro aspecto estudiado fue la identificación y combate del complejo de microorganismos Fusarium y Rhizoctonia que participan en el ahogamiento (damping-off) de las plántulas; enfermedad que afecta la producción de especies forestales en viveros (Gómez y Yáñez, 1963; Gómez, 1970); además de, las enfermedades que dañan a Pinus radiata D. Don (Salinas y Gómez, 1975). Los resultados de los diversos estudios sobre 
enfermedades forestales derivaron en la publicación del primer catálogo de la colección de cultivos de hongos del INIF (Salinas, 1982).

\section{Aportaciones del INIFAP}

\section{Contribución en materia de plagas forestales}

Durante los primeros años a partir de la creación del INIFAP, la investigación en sanidad forestal siguió la inercia propia del instituto antecesor. Jaime Villa Castillo retomó como línea importante de investigación, el uso de semioquímicos para la atracción y el monitoreo de los insectos descortezadores: D. mexicanus y $D$. adjunctus en Jalisco, y demostró que la frontalina y alfa-pineno atraían de manera significativa a las dos especies; además, definió los picos de vuelo para ambas (Villa, 1992a).

Respecto al control químico de insectos descortezadores, el citado investigador experimentó en campo el efecto de la fumigación con fosfamina en trozas envueltas con plástico; sus resultados indican, que si bien con ese plaguicida se logró la mortalidad de los descortezadores, de forma colateral ocurrió la muerte de sus enemigos naturales; aspecto crítico que se debe considerar en el control químico de plagas forestales (Villa, 1992b).

Otra aportación del INIFAP en sus inicios la hizo Blas Enrique Díaz Ortiz, quien realizó uno de los estudios más completos sobre los insectos barrenadores de conos y semillas en las coníferas de la Sierra de San Pedro Mártir, Baja California. En dicho trabajo se determinaron 16 taxones y su impacto en la producción de semillas; se concluyó que, en términos generales, el daño causado por este grupo de insectos es somero y no la causa principal de la escasa regeneración natural que presentaba Pinus spp. y Abies concolor (Gordon) Lindl. ex Hildebr en ese ecosistema. Entre los barrenadores de conos y semillas más frecuentes se descubrieron y describieron diversas especies de los géneros Conophthorus Hopkins, Earomyia Zetterstedt, Cydia Hübner, Dioryctria Zeller y Argyresthia Hübner (Díaz, 1988a; 1988b). 
Para el estado de Chihuahua, a dos años de la creación del INIFAP, Raúl Narváez Flores detectó en 18 localidades, ubicadas en los municipios Bocoyna y Guerrero, la presencia de Cydia phyllisi Miller; barrenador de los conos de Picea chihuahuana Martínez, conífera en peligro de extinción. Narváez (1988) registró un promedio de afectación de $85 \%$ en los conos, $21 \%$ en semillas y $37 \%$ en semillas vanas. Además, definió el ciclo de vida de esta palomilla y sus tiempos de desarrollo; con ello, determinó el periodo adecuado (octubre a abril) para realizar su control mecánico, consistente en la recolecta y quema de los conos infestados que caen al suelo.

A partir del año 2002, en el INIFAP se dio un fuerte impulso al estudio de plagas y enfermedades forestales, como resultado de la creación del Fondo Sectorial Para la Investigación e Innovación Tecnológica Forestal, conformado por la Comisión Nacional Forestal (Conafor) y el Consejo Nacional de Ciencia y Tecnología (Conacyt), el cual se sumó al financiamiento de instituciones de ámbito estatal. Así, varios investigadores del INIFAP atendieron las demandas de investigación de dicho fondo, y plantearon la solución de problemas con el enfoque del manejo integrado de plagas, cuyo fin es proponer alternativas ecológicamente aceptables.

En este contexto sobresalen los trabajos de Alfredo Sánchez Salas, Luis Mario Torres Espinoza, Antonio Cano Pineda y Oscar Ulises Martínez Burciaga, investigadores del Campo Experimental Saltillo, quienes Ilenaron en parte el vacío de conocimiento sobre las especies de descortezadores de coníferas presentes en Coahuila y Nuevo León (Sánchez et al., 2003); definieron los periodos de vuelo y ataque de Dendroctonus pseudotsugae Hopkins, D. adjunctus y Dendroctonus brevicomis LeConte en la Sierra de Arteaga, y los tratamientos convenientes para su control (Torres et al., 2004).

Es importante citar los estudios de los investigadores del Centro de Investigación Regional Norte-Centro y del Centro de Investigación Regional Pacífico Centro, quienes determinaron los periodos de vuelo de diversos taxones de descortezadores en Chihuahua, Durango, Coahuila, Jalisco, Michoacán y Aguascalientes; cuyos aportes fueron las bases para el monitoreo de estos insectos a través del uso de semioquímicos (Sánchez et al. 2007, Vázquez et al., 2007). 
En tiempos más recientes, Sánchez et al. (2012a) validaron el efecto de la feromona antiagregante $\mathrm{MCH}$ para la prevención e interrupción del ataque de $D$. pseudotsugae sobre Pseudotsuga menziesii (Mirb.) Franco en Durango y Chihuahua, lo cual constituye una tecnología no contaminante para la prevención y control de los brotes del insecto en cuestión. Rivera-Dávila et al. (2021) demostraron que las feromonas de antiagregación $\mathrm{MCH}$ y verbenona son, prácticamente, no tóxicas para los organismos que habitan el bosque, suelo y agua, por lo que son compuestos volátiles adecuados para prevenir el ataque de $D$. pseudotsugae en el primer caso, y para especies del complejo frontalis en el segundo.

Durante la última década, en algunas investigaciones realizadas en el INIFAP sobre ecología química de insectos se han determinado los semioquímicos adecuados para el monitoreo y trampeo masivo de $D$. frontalis y $D$. mexicanus en la Sierra Gorda de Querétaro (Sánchez et al., 2017; Sánchez-Martínez y Reséndiz-Martínez, 2020).

La adopción de tecnologías generadas en el Instituto sobre insectos descortezadores se refleja en el contenido de la Norma Oficial Mexicana NOM-019-SEMARNAT-2017 (Semarnat, 2018), en la que se establecen los lineamientos para el monitoreo, la prevención y el combate de insectos descortezadores de coníferas en México; y en la cual se utiliza como fundamentos técnicos varias de las publicaciones generadas por investigadores del INIFAP (Semarnat, 2018).

En el INIFAP, también se han buscado soluciones para los problemas fitosanitarios presentes en áreas naturales protegidas, donde los métodos convencionales de control mecánico-químico con frecuencia tienen limitaciones legales y de índole sociocultural. En estos casos, se han retomado los métodos de inyección de árboles, pero con tecnología que minimiza las heridas en los puntos de inyección y se evita la exposición de insecticidas fuera de la corteza del árbol, para no contaminar el ambiente (Espinosa et al., 2014; Sánchez et al., 2014; Gochez-López et al., 2018).

Otra contribución importante del INIFAP se refiere al monitoreo de plagas de interés cuarentenario, como fue el caso de la conchuela del eucalipto (Glycaspis 
brimblecombei Moore) y la cochinilla rosada del hibisco (Maconellicoccus hirsutus Green), insectos exóticos que constituyeron demandas de investigación del Fondo Conafor-Conacyt en la primera década del presente siglo.

En el caso de G. brimblecombei, Sánchez y González (2006) desarrollaron una metodología para la detección, reproducción y aumento de las poblaciones de Psyllaephagus bliteus Riek, parasitoide natural de dicho psílido. En cuanto a $M$. hirsutus, insecto que afecta las plantaciones de Tectona grandis L. f. y diversas plantas silvestres y ornamentales, González-Gaona et al. (2010) validaron la efectividad de las feromonas sexuales de este insecto, identificadas y sintetizadas por Zhang et al. (2004) y Zhang y Amalin (2005); mapearon su distribución geográfica en la región Pacífico de México; identificaron a sus principales hospederos y proporcionaron fundamentos científicos para su control biológico.

Respecto a otros grupos de insectos de valor forestal, destaca la aportación del INIFAP en el estudio de las moscas sierra de las coníferas (Hymenoptera: Diprionidae), así como de insectos barrenadores de encinos y coníferas antes desconocidos. En el primer caso, se contribuyó en la descripción de la nueva especie Zadiprion ojedae Smith \& Sánchez-Martínez; la actualización de la clave taxonómica del género Zadiprion Rohwer (Smith et al., 2012); la descripción y biología de la nueva especie de Monoctenus sanchezi Smith (Smith et al., 2010); el conocimiento de la biología de Neodiprion autumnalis y Zadiprion ojedae (Sánchez et al., 2012b); y la actualización del conocimiento sobre la identificación y distribución de todos los taxones de mosca sierra de coníferas hasta ahora conocidos en México (Coria-Avalos et al., 2014; González y Sánchez, 2018).

Actualmente, la Comisión Nacional Forestal considera a los investigadores del Campo Experimental Pabellón y del Centro Nacional de Investigación Disciplinaria en Conservación y Mejoramiento de Ecosistemas Forestales (Cenid Comef) como referentes para la capacitación de su personal técnico sobre insectos descortezadores y moscas sierra de coníferas. Igualmente notoria en estos centros de investigación ha sido la identificación, distribución y control del barrenador de encinos Megapurpuricenus magnificus LeConte 
(antes Crioprosopus magnificus) (Sánchez-Martínez et al., 2010a; Sánchez et al., 2014); la identificación y aspectos biológicos del barrenador de pinos Sirex obesus Bradley; y la identificación del descortezador del ahuehuete (Taxodium mucronatum Ten.) Eudociminus mannerheimii Boheman (Sánchez-Martínez et al., 2010b).

\section{Contribución en materia de enfermedades}

En el INIFAP la investigación se ha enfocado, principalmente, en el estudio de las enfermedades forestales que afectan a los bosques naturales y a los bosques urbanos; en este último caso, con especial interés en los de la Ciudad de México (Reséndiz et al., 2019).

El arbolado público se considera un elemento esencial dentro las áreas urbanas por los múltiples beneficios socioculturales y ambientales que aporta: el valor estético y provisión de sombra en los espacios de esparcimiento; su función en atenuar los escurrimientos fluviales; y su acción como mejorador de la calidad del aire, entre otros (Urcelay et al., 2012).Al respecto, Francisco Reséndiz Martínez y Rodolfo Salinas Quinard continuaron con el estudio de los patógenos que afectan a Pinus radiata; la micoflora que incide en Abies religiosa (Kunth) Schltdl. et Cham. del Parque Cultural y Recreativo Desierto de los Leones en la Ciudad de México y sobre una posible nueva roya que afecta a Pinus montezumae Lamb. (Reséndiz y Salinas, 1989; Salinas, 1989).

Salinas Quinard también aportó conocimientos sobre el ciclo de vida, signos y daños de la roya Cronartium conigenum Hedgc. \& N. R. Hunt en pinos de la Sierra Madre Oriental, Sierra Madre Occidental y sureste de México, en colaboración con investigadores representantes de la Comisión Forestal para América del Norte (Peterson y Salinas, 1967; Sutherland et al., 1987).

En tiempos más recientes, investigadores del Cenid Comef han continuado los estudios de la condición sanitaria de las áreas verdes de la Ciudad de México. Por ejemplo, Velasco et al. (2002) registraron mayor incidencia de plagas (9 a $19 \%$ ) que enfermedades (4 a $14 \%$ ) en los bosques del Distrito Federal; en los cuales Pinus hartwegii Lindl. era 
la especie más atacada por plagas, con $8.7 \%$ y por enfermedades con $7.6 \%$. En ese estudio fue importante el hallazgo de que la plaga principal no era un insecto, sino la ardilla Sciurus sp., y que la enfermedad más importante era la del follaje causada por el hongo: Lophodermium sp.

En una investigación detallada sobre muérdagos verdaderos en 16 alcaldías de la Ciudad de México, Arriola et al. (2012) registraron a Cladocolea loniceroides (Van Tieghem) Kuijt, Phoradendron velutinum (DC.) Oliv. y Struthanthus interruptus (Kunth.) BI. como las plantas parásitas más importantes que estuvieron presentes en ocho alcaldías, con porcentajes de infestación inferiores a $5 \%$, en la mayoría de los casos; y señalaron que pueden mantenerse bajo control, mediante podas mecánicas.

Velasco et al. (2013) hicieron un inventario completo de los árboles del Bosque de San Juan de Aragón, Cd. de México, que incluyó la evaluación de la condición sanitaria y de otras variables que interactúan con la salud de los árboles y el bosque urbano en su conjunto; entre ellas, la especie, edad, vigor y daños mecánicos. Sus resultados indicaron que, a pesar de registrar una baja diversidad de especies, la condición general del Bosque de San Juan de Aragón se clasificó como buena, y se recomendaron podas para alrededor de 5200 árboles que en su momento lo requerían.

En esta línea de investigación, Reséndiz et al. (2015) determinaron los agentes de enfermedades foliares de los árboles del Parque Cultural y Recreativo Tezozómoc en Azcapotzalco; y Gutiérrez et al. (2019) identificaron los agentes que afectan a la vegetación en la Segunda Sección del Bosque de Chapultepec.

Con referencia a las enfermedades presentes en bosques naturales, Martínez et al. (2014) estudiaron el efecto del muérdago enano Arceuthobium vaginatum (Willd.) Presl. subsp. vaginatum en Pinus hartwegii del Parque Nacional Volcán Nevado de Colima, y concluyeron que la infestación por muérdago enano redujo $22 \%$ del crecimiento en altura, $9 \%$ en diámetro y $50 \%$ en volumen. Además, precisaron que esta planta parásita afectó en porcentajes similares al incremento corriente anual e incremento medio anual, lo cual disminuye el volumen de madera producido y aumenta el turno técnico maderable del arbolado, en un promedio de 10 años. Con 
base en ello, recomendaron la aplicación de prácticas de prevención y combate del parásito, así como el establecimiento de sitios de monitoreo continuo.

Coria et al. (2010) experimentaron sobre el efecto de la tierra de diatomeas y 2cloroetil fosfórico sobre Arceuthobium globosum Hawksworth \& Wiens subsp. grandicaule en Pinus pseudostrobus Lindl. Los tratamientos tuvieron el mismo impacto de supresión sobre la parte aérea de Arceuthobium globosum ssp. grandicaule cuando se usó tierra de diatomeas. La muerte del área foliar sobrevino a los 15 días y la caída de la parte aérea a los 45. La mayor eficacia de este producto ocurrió al $7.5 \%$ en $200 \mathrm{~L}$ de agua, y ninguna dosis produjo síntomas de fitotoxicidad a la planta hospedera.

En cuanto a estudios de enfermedades en plantaciones comerciales, las contribuciones del INIFAP han sido más modestas; sin embargo, se ha generado información básica y de diagnóstico. Por ejemplo, Bernal et al. (2009) determinaron al patógeno Chrysoporthe cubensis (Bruner) Gryzenhout \& M. J. Wingf. como el causante del cancro en plantaciones de eucalipto en Chiapas y Oaxaca. La información sobre el agente causal es relevante porque hasta $70 \%$ de los árboles son dañados por este hongo, lo cual incide en la producción de las plantaciones comerciales.

Gijón-Hernández et al. (2019) determinaron que el agente causal de lesiones necróticas en hojas nuevas, brotes y tallos en el cultivo de hule [Hevea brasiliensis (Willd. ex A. Juss.) Müll. \& Arg.] corresponde al complejo Colletotrichum gloesporioides (Penz.) Penz. y Sacc., y no a Microcyclus ulei (Henn.) Arx. al que los productores de hule de San Juan Bautista, Tuxtepec, Oaxaca atribuían los daños. Estas precisiones en la correcta identificación de los agentes patógenos mediante técnicas tradicionales y moleculares son básicas para implementar las medidas correctas de control de las enfermedades forestales. 


\section{Perspectivas y retos}

La investigación sobre plagas y enfermedades realizada por el INIFAP se ha enfocado desde una perspectiva de sanidad forestal, al considerar a los organismos plaga como los causantes directos de los daños; con ello, se ha contribuido a la identificación taxonómica y, en algunos casos, al diseño de medidas concretas para el control directo o el manejo integral de los agentes causales. Sin embargo, en las últimas décadas del siglo XX surgió el concepto "salud forestal", el cual es más amplio y tiene una perspectiva ecológica. Bajo esta última acepción, si bien se reconoce el daño económico que algunos organismos provocan en la producción forestal, también se valoran las funciones que desempeñan en los diversos procesos ecológicos, tales como el flujo de energía y la sucesión vegetal, entre otros (Kolb et al., 1991; Mistretta, 2002; Raffa et al., 2008).

El concepto de salud forestal, aunque ya reconocido, ha sido poco adoptado en la investigación hasta ahora realizada en el INIFAP; no obstante, es necesario incorporarlo para lograr identificar los factores tanto inmediatos, como de fondo que propician comportamientos inusuales en los seres vivos para actuar como plaga.

Un bosque saludable, desde el punto de vista ecológico, es aquella condición en la cual, para el propio funcionamiento del ecosistema, ocurren disturbios naturales, pero bajo un umbral que permite la resiliencia e impide una pérdida permanente de la vegetación forestal. Asimismo en un contexto utilitario, un bosque saludable es aquel que aun con la presencia de disturbios naturales provee productos y servicios ecosistémicos forestales para bien de la sociedad (Kolb et al., 1991; Millar y Stephenson, 2015).

Una tendencia reciente de investigación en materia de salud forestal internacional se refiere al estudio del impacto del cambio climático en el comportamiento de insectos y patógenos que inciden directamente en la vitalidad de los bosques (Moore y Allard, 2009). Varios especialistas afirman que el cambio climático ya está afectando el desarrollo biológico y la dinámica poblacional de insectos plaga en varias partes del mundo (Bentz et al., 2010; Allen et al., 2010). Existen casos recientes de comportamientos extraños de insectos y 
patógenos que han causado una mortalidad sin precedente de millones de árboles en millones de hectáreas (Allen et al., 2010; Bentz et al., 2010).

Ante este fenómeno, algunos investigadores, pronostican el aumento de generaciones por año de insectos plaga y la colonización de especies en nuevas zonas geográficas (Williams y Liebhold, 2002; Carroll et al., 2003; Bentz et al. 2010). También, se pronostica mayor actividad de los patógenos que provocan enfermedades forestales (Sturrock et al., 2011).

En México, en la última década se han presentado brotes epidémicos atípicos de insectos descortezadores y defoliadores (del-Val y Sáenz-Romero, 2017; González y Sánchez, 2018; Cervantes-Martínez et al., 2019), así como enfermedades que originan declinación y defoliación (Pérez et al., 2017); no obstante, la investigación específica sobre el cambio climático como factor causal o contribuyente del comportamiento atípico de insectos y patógenos es incipiente en el país; y ante tal necesidad, existe un reto para el INIFAP de innovar en la forma de abordar el tema, a partir del concepto de salud forestal y de los escenarios de cambio climático.

Hasta ahora han sido poco exploradas las interacciones entre la abundancia de los organismos considerados plaga y el estado de los bosques desde el punto de vista de composición de especies, estructura, estado de desarrollo del rodal o plantaciones; variables que influyen en el comportamiento e impacto de insectos y patógenos, y en la resiliencia de bosques, plantaciones y árboles urbanos. Es insuficiente modelar la distribución geográfica de los organismos plaga y su impacto, con tan solo variables climáticas y escenarios teóricos, pues a escala local, los efectos del cambio climático son influenciados por otros factores intrínsecos del bosque, como la diversidad de especies, la edad de los hospederos, la densidad de los rodales y la complejidad en la estructura forestal (Bentz y Jönsson, 2015; Morin et al., 2018).

Con base en estudios que aborden estos aspectos de investigación, el INIFAP estará en posibilidad de aportar no solo diagnósticos y medidas de control directo, sino recomendaciones y estrategias orientadas a la prevención de brotes epidémicos, bajo 
la premisa de que el cambio climático está ocurriendo. Para ello, ante las limitaciones económicas que los tiempos están imponiendo a la investigación forestal en México, es necesario que en el INIFAP se retome y fortalezcan los lazos de cooperación de intercambio técnico y científico que se han establecido a lo largo de los años con instituciones nacionales y de otros países.

\section{Agradecimientos}

Los autores agradecen al Dr. Luis Ángel Rodríguez del Bosque y al Dr. Rogelio Flores Velázquez por la invitación a realizar este artículo de revisión.

\section{Conflicto de intereses}

Los autores declaran no tener conflicto de interés alguno con esta publicación.

\section{Contribución por autor}

Guillermo Sánchez Martínez: investigación bibliográfica sobre plagas forestales y elaboración de manuscrito; José Francisco Reséndiz Martínez: investigación bibliográfica sobre enfermedades forestales y elaboración de manuscrito.

\section{Referencias}

Allen, C. D., A. K. Macalady, H. Chenchouni, D. Bachelet, N. McDowell, M. Vennetier, T. Kitzberger, A. Rigling, D. D. Breshears, E. H. (Ted) Hogg, P. Gonzalez, R. Fensham, Z. Zhang, J. Castro, N. Demidova, J. H. Lim, G. Allard, S. W. Running, A. Semerci and N. Cobb. 2010. A global overview of drought and heat-induced tree mortality reveals emerging climate change risks for forests. Forest Ecology and Management 259: 660-684. Doi: 10.1016/j.foreco.2009.09.001. 
Arriola P., V. J., E. Velasco B., T. Hernández T., A. González H., y M. E. Romero S. 2012. Los muérdagos verdaderos del arbolado de la Ciudad de México Revista Mexicana de Ciencias Forestales 4 (19): 34-45. Doi: 10.29298/rmcf.v4i19.377.

Bentz, B. J., J. Régnière, C. J. Fettig, E. M. Hansen, J. L. Hayes, J. A. Hicke, R.G. Kelsey, J. F. Negrón and S. J. Seybold. 2010. Climate change and bark beetles of the western United States and Canada: Direct and indirect effects. Bioscience 60 (8): 602-613. Doi:10.1525/bio.2010.60.8.6.

Bentz, B. J. and A. M. Jönsson. 2015. Modeling bark beetle responses to climate change. In: Vega, F. E. and R. W. Hofstetter (eds.). Bark beetles: Biology and ecology of native and invasive species. Elsevier. San Diego, CA., USA. pp. 533-553.

Bernal L., I., C. J. Palacios J., R. Santos G., L. Vázquez S., H. A. Zavaleta M., H. S, Azpiroz R., C. Parraguirre L. y M. del P. de la Garza L. de L. 2009. Identificación del agente patógeno del cancro del eucalipto en plantaciones del Sureste de México. Ciencia Forestal en México 34 (105): 19-37. https://cienciasforestales.inifap.gob.mx (15 de marzo de 2021).

Carroll, A. L., S. W. Taylor, J. Regniere and L. Safranyik. 2003. Effect of climate change on range expansion by the mountain pine beetle in British Columbia. The Bark Beetles, Fuels, and Fire Bibliography. Utah State University. Logan, UT, USA. Paper 195. pp. 223-232. https://digitalcommons.usu.edu/barkbeetles/195 (12 de marzo de 2021).

Castro C., J. 1981. Contribución al estudio de la biología del defoliador de pino Neodiprion fulviceps (Cresson) complex (Hymenoptera: Diprionidae) en el estado de Chihuahua. Ciencia Forestal 6(30): 43-51.

Cisneros P., S. 1970. Observaciones sobre Zadiprion vallicola Rohwer en la meseta tarasca, Michoacán. INIF. México, D.F., México. Boletín Técnico No. 33. 14p. 
Cervantes-Martínez, R., J. Cerano-Paredes, G. Sánchez-Martínez, J. Villanueva-Díaz, G. Esquivel-Arriaga, V. H. Cambrón-Sandoval, J. Méndez-González and L. U. Castruita-Esparza. 2019. Historical bark beetle outbreaks in Mexico, Guatemala and Honduras (1895-2015) and their relationship with droughts. Revista Chapingo Serie Ciencias Forestales y del Ambiente 25 (2): 269-290. Doi: 10.5154/r.rchscfa.2019.01.006.

Comisión Interamericana de Ambiente y Desarrollo (CCAD). 2017. Estrategia regional de salud y sanidad forestal para Centroamérica y República Dominicana. SICA-CCAD. El Salvador, San Salvador. 96 p. https://www.sica.int/documentos/publicacion-estrategia-regional-de-salud-y-sanidad-forestalpara-centroamerica-y-republica-dominicana-2016-2026_1_124498.html (10 de mayo de 2021).

Coria Á., V.M., I. Vázquez C., H. J. Muñoz F. y J. Villa C. 2010. Impacto de tierra de diatomeas sobre Arceuthobium globosum Hawksworth \& Wiens subsp. grandicaule en Pinus pseudostrobus Lindl. Revista Mexicana de Ciencias Forestales 1 (1): 39-46. Doi: $10.29298 /$ rmcf.v1i1.651.

Coria-Avalos., V. M., E. González-Gaona, A. Pulido-Herrera y H. J. Muñoz-Flores. 2014. Detección y descripción morfológica de "mosca sierra" en bosques de la comunidad indígena de Nuevo San Juan Parangaricutiro, Michoacán, México. Entomología Mexicana 1: 426-430. http://www.entomologia.socmexent.org/revista/2014/EC/078.pdf (12 de marzo de 2021).

del-Val, E. y C. Sáenz-Romero. 2017. Insectos descortezadores (Coleoptera:Curculionidae) y cambio climático: problemática actual y perspectivas en los bosques templados. Tip Revista Especializada en Ciencias Químico-Biológicas 20 (2): 53-60. Doi: 10.1016/j.recqb.2017.04.006.

Díaz O., B. E. 1988a. Biología del barrenador de semillas de coníferas Cydia miscitata Heinrich en la Sierra de San Pedro Mártir, Baja California. Ciencia Forestal 13 (63): 15-27. 
Díaz O., B. E. 1988b. Insectos que afectan a conos y semillas en la Sierra de San Pedro Mártir, Baja California. Ciencia Forestal 13 (63): 29-70.

Espinosa F., N., V. J. Arriola P., V. Guerra de la C., V. Cibrián L. y G. Galindo F. 2014. Control de plagas en conos y semillas de Pseudotsuga menziesii (Mirb.) Franco mediante insecticidas sistémicos. Revista Mexicana de Ciencias Forestales 5(23): 30-41. Doi: 10.29298/rmcf.v5i23.340.

Gijón-Hernández, A. R., I. M. Pérez-Gálvez, B. Torres-Huerta, H. V. Rojas-Silva y E. Ortiz C. 2019. Identificación del agente causal de la antracnosis en el cultivo de hule [Hevea brasiliensis (Willd. ex A. Juss.) Müll. Arg.]. Revista Mexicana de Ciencias Forestales 10 (56): 136-148. Doi: 10.29298/rmcf.v10i56.551.

Gochez-López, E., V. J. Arriola-Padilla, A. Perea-Alcalá, J. F. Reséndiz-Martínez y A. D. Camacho. 2018. Insecticidas sistémicos para el control de Dendroctonus adjunctus Blandford, 1987 en el Nevado de Toluca. Revista Mexicana de Ciencias Forestales 6 (27): 50-63. Doi: 10.29298/rmcf.v6i27.280.

Gómez N., M .S. y O. Yáñez M. 1963. Damping off en Pinus montezumae Lamb. y su combate. Secretaría de Agricultura y Ganadería. México, D.F., México. Boletín Técnico No. 7. 31 p.

Gómez N., M. S., R. Echenique M. y R. Salinas Q. 1969. Índices de laboratorio sobre resistencia de la madera a la pudrición en once especies forestales mexicanas. INIF. México, D.F., México. Boletín Técnico No. 31.40 p.

Gómez N., M. S. 1970. Microorganismos asociados con "Damping-off" en plántulas de Disocorea composita Hemsl. INIF, México D.F., México. Boletín Técnico No. 36.12 p. 
González G., E. y G. Sánchez M. 2018. Identificación y manejo de moscas sierra de la familia Diprionidae presentes en el centro norte de México. Conacyt-Conafor. Zapopan, Jal., México. Folleto Técnico s/n. 122 p.

http://sivicoff.cnf.gob.mx/ContenidoPublico/09\%20Manuales\%20t\%C3\%A9cnicos/M anual\%20moscas\%20sierra.pdf (13 de marzo de 2021).

González-Gaona, E., G. Sánchez-Martínez, A. Zhang, J. Lozano-Gutiérrez y F. CarmonaSosa. 2010. Validación de dos compuestos feromonales para el monitoreo de la cochinilla rosada del hibisco en México. Agrociencia 44 (1): 65-73. https://www.agrocienciacolpos.mx/index.php/agrociencia/article/view/776 (13 de marzo de 2021).

Gutiérrez G., M. y R. Muñiz V. 1984. La situación de las plagas en el Bosque de Chapultepec de la Ciudad de México. INIF-SARH. México, D.F., México. Boletín Técnico No. 100. 41 p.

Gutiérrez R., M., D. Ruiz J., J. F. Reséndiz M., L. I. García D. y M. Ramón M. 2019. Salud forestal del arbolado de la segunda sección del bosque de Chapultepec de la Ciudad de México. Sociedades rurales, Producción y Medio Ambiente 37 (37): 137164. https://publicaciones.xoc.uam.mx/ (12 de mayo de 2021).

Instituto Nacional de Investigaciones Forestales (INIF). 1982. 50 años de investigación forestal en México. INIF. México, D.F., México. Publicación especial No. 39. 77p.

Islas S., F. 1968. Observaciones Biológicas sobre un descortezador de pinos:

Dendroctonus adjuntus Bldf.- Col. Scolytidae. Secretaría de Agricultura y Ganadería. México, D.F., México. Boletín Técnico No.25. 21 p.

Islas S., F. 1980a. Observaciones sobre la biología y el combate de los escarabajos descortezadores de los pinos: Dendroctonus adjunctus Blf; D. mexicanus Hpk. y D. frontalis Zimm., en algunas regiones de la república mexicana. INIF. México, D.F., México. Boletín Técnico Núm. 66. 38 p. 
Islas S., F. 1980b. El descortezador suriano de los pinos ( $D$. frontalis Z.) y la lucha biológica. Ciencia Forestal 5(28): 57-64.

Islas S., F y R. Muñiz V. 1985. Morfología de Zadiprion vallicola Roh. (Hymenoptera: Diprionidae). Ciencia Forestal 10 (57): 3-14.

Kolb, T. E., M.R. Wagner and W. W. Covington. 1991. Forest health from different perspectives. Technical Report RM-GTR-267. USDA Forest Service, Fort Collins, CO, USA. pp. 5-13. http://openknowledge.nau.edu/id/eprint/2573 (15 de marzo de 2021).

Martínez S., M., S. Madrigal H., I. Vázquez C., E. Velasco B., C. R. Morales N. y F. Villareal G. 2014. Efecto de Arceuthobium vaginatum (Willd.) Presl. subsp. vaginatum en Pinus hartwegii Lindl., en Colima. Revista Mexicana de Ciencias Forestales 6 (29): 44-55. Doi: 10.29298/rmcf.v6i29.215.

Mayo J., P. 1976. Observaciones preliminares sobre la biología y hábitos de Neodiprion sp. circa gillettei Rohwer. Secretaría de Agricultura y Ganadería. México, D.F., México. Boletín Técnico No. 48. 17 p.

Millar, C. I. and N. L. Stephenson. 2015. Temperate forest health in an era of emerging megadisturbance. Science 3499(6250): 823-826. Doi: 10.1126/science.aaa9933.

Mistretta, P. A. 2002. Southern Forest Resource Assessment highlights: Managing for forest health. Journal of Forestry 100(7):24-27. Doi: 10.1093/jof/100.7.24.

Moore, B. y G. Allard. 2009. Los impactos del cambio climático en la sanidad forestal. FAO. Documento de trabajo FBS/34S. Roma, Italia.42 p.

Morin, X., L. Fahse, H. Jactel, M. Scherer-Lorenzen, R. García-Valdés and H. Bugmann. 2018. Long-term response of forest productivity to climate change is mostly driven by change in tree species composition. Scientific Reports 8: 5627. Doi: $10.1038 / s 41598-018-23763-y$. 
Narváez F., R. 1988. Cydia phyllisi (Lepidoptera: Tortricidae) gusano barrenador de la semilla de Picea chihuahuana Martínez especie en peligro de extinción. In: González V., C. E., A. B. Villa S. y M. de C. Pineda T. (eds.). Memoria del IV Simposio Nacional de Parasitología Forestal y IV Reunión de Plagas y Enfermedades Forestales. SARH-INIFAP-Dirección General de Sanidad y Protección Agropecuaria y Forestal-Sociedad Mexicana de Entomología. Ciudad, estado, México. Publicación Especial Núm. 59. Tomo I. pp. 375-386.

Organización de las Naciones Unidas para la Agricultura y la Alimentación (FAO). 2018. El estado de los bosques del mundo. http://www.fao.org/state-of-forests/es/ (15 de marzo de 2021).

Pérez O., M.P. y R. Salinas Q. 1977. Prueba rápida de laboratorio indicadora de resistencia a pudrición de dos especies de encinos. Ciencia Forestal 6(2): 3-19.

Pérez M., R., M.E. Romero S., A. González H., E. Pérez S. y V. J. Arriola P. 2017. Distribución potencial de Lophodermium spp., en bosques de coníferas, con escenarios de cambio climático. Revista Mexicana de Ciencias Forestales 7 (36): 81-97. Doi: 10.29298/rmcf.v7i36.61.

Perusquía O., J. 1978. Descortezador de los pinos Dendroctonus spp. Taxonomía y Distribución. SARH, DGICF. México D.F. México. Boletín Técnico No. 55. 31 p.

Perusquía O., J. 1982. Insectos asociados a los descortezadores Dendroctonus spp. de los pinos. Instituto Nacional de Investigaciones Forestales. México, D.F., México. Boletín técnico Núm. 83. pp. 5-7.

Peterson, R. S. y R. Salinas Q. 1967. Cronatium conigenum: Distribución y efectos en los pinos. INIF, México, D.F., México. Boletín Técnico No. 19. 11p. 
Raffa, K. F., B. H. Aukema, B. J. Bentz, A. L. Carroll, J. A. Hicke, M. G. Turner and W. H. Romme. 2008. Cross-scale drivers of natural disturbances prone to anthropogenic amplification: the dynamics of bark beetle eruptions. Bioscience 58(6): 501-517. Doi: 10.1641/B580607.

Reséndiz M., J. F. y R. Salinas Q. 1989. Observaciones preliminares sobre la micoflora asociada a Abies religiosa (H.B.K.) Schl. et Cham. In: González V., C. E., A. B. Villa S. y M. de C. Pineda T. (eds.) Memorias del IV Simposio Nacional sobre Parasitología Forestal. Tomo II. México, D. F., México. Publicación especial No. 60. pp. 695-701.

Reséndiz M., J. M., L. Guzmán D., A. L. Muñoz V., C. Nieto de P. P. y L. P. Olvera C. 2015. Enfermedades foliares del arbolado en el Parque Cultural y Recreativo Tezozómoc, Azcapotzalco, Distrito Federal. Revista Mexicana de Ciencias Forestales 6 (30): 106-123. Doi: 10.29298/rmcf.v6i30.211.

Reséndiz M., J. F., L. Guzmán D., A. L. Muñoz V., P. Olvera C., M. de L. Pacheco H. y V. J. Arriola P. 2019. Insectos y ácaros fitófagos del arbolado en el Parque Recreativo y Cultural Tezozómoc, Azcapotzalco, Ciudad de México. Revista Mexicana de Ciencias Forestales 10 (56): 149-173. Doi: 10.29298/rmcf.v10i56.454.

Rivera-Dávila, O. L., G. Sánchez-Martínez and R. Rico-Martínez. 2021. Ecotoxicity of pesticides and semiochemicals used for control and prevention of conifer bark beetle (Dendroctonus spp.) outbreaks. Chemosphere 263:128375.

Doi: $10.1016 /$ j.chemosphere.2020.128375.

Salinas Q., R. y Gómez N., M. S. 1975. Enfermedades del Pinus radiata D. Don. INIF, México, D. F., México. Nota Técnica No. 8. 20 p.

Salinas Q., R. y M. G. Macías C. 1979. Técnicas anatomo- microscópicas para detectar microorganismos asociados con descortezadores (Dendroctonus spp.) productores de la "Mancha azul". Ciencia Forestal 20(4): 3-12. 
Salinas Q., R. 1982. Enfermedades forestales en México. Ciencia Forestal 7 (35): 21-30.

Salinas Q., R. 1989. Informe complementario sobre una posible nueva roya de pinos. In: González V., C. E., A. B. Villa S. y M. de C. Pineda T. (eds.) Memorias del IV Simposio Nacional sobre Parasitología Forestal. Tomo II. SARH-INIFAP-Dirección General de Sanidad y Protección Agropecuaria y Forestal-Sociedad Mexicana de Entomología. México, D. F., México. Publicación especial No. 60. pp. 625-630.

Sánchez M., G. y E. González G. 2006. Manual para el control biológico del psílido del eucalipto (Glycaspis brimblecombei Moore). INIFAP-CIRNOC, Campo Experimental Pabellón. Pabellón de Artega, Ags., México. Folleto Técnico Núm. 28. 47 p.

Sánchez M., G., L. M. Torres E., I. Vázquez C., E. González G. y R. Narváez F. 2007. Monitoreo y manejo de insectos descortezadores de coníferas. INIFAP-CIRNOC, Campo Experimental Pabellón. Pabellón de Artega, Ags., México. Libro Técnico Núm. 4. 107 p.

Sánchez-Martínez, G., A. Equihua-Martínez, E. González-Gaona and R. W. Jones. 2010a. First record of Eudociminus mannerheimii (Boheman) (Coleoptera:

Curculionoidea) attacking Taxodium mucronatum Ten. (Cupressaceae) in Jalisco, Mexico. The Coleopterists Bulletin 64 (1): 96-97. Doi: 10.1649/0010-065x-64.1.96.

Sánchez-Martínez, G., O. Moreno-Rico and M. E. Siqueiros-Delgado. 2010b. Crioprosopus magnificus LeConte (Coleoptera: Cerambycidae) in Aguascalientes, Mexico: Biological observations and geographical distribution. The Coleopterists Bulletin 64(4): 319-328. Doi: 10.1649/0010-065x-64.4.319.

Sánchez M., G., C. J. Mehmel, N. E. Gillette, E. González G., J. A. López H., J. C. Monárrez G., J. L. García Rodríguez, S. R. Mori, H. E. Alanís Morales, J. M. Mejía B., M. Cano R., M. A. Cortés C. y L. M. Torres E. 2012a. Fundamentos para el control integral del descortezador Dendroctonus pseudotsugae barragani Furniss en México. INIFAP-CIRNOC, Campo Experimental Pabellón. Pabellón de Artega, Ags., México. Folleto Técnico Núm. 46. 54 p. 
Sánchez M., G., H. E. Alanís, M., M. Cano R. y J. A. Olivo M. 2012b. Biología de y aspectos taxonómicos de dos especies de moscas sierra de los pinos en Chihuahua. INIFAP-CIRNOC-Campo Experimental Pabellón. Pabellón de Artega, Ags., México Folleto Técnico Núm. 44. 26 p.

Sánchez M., G., O. Moreno R., J. F. Reséndiz M., V. J. Arriola P. y G. González G. E. 2014. El barrenador de los encinos Crioprosopus magnificus. Bases para su diagnóstico y control. INIFAP-CIRNOC-Campo Experimental Pabellón. Pabellón de Artega, Ags., México. Folleto Técnico. Núm. 57. 26 p.

Sánchez M., G., J. F. Reséndiz M. y S. Santana E. 2017. Fundamentos para el uso de semioquímicos en el manejo integral de insectos descortezadores de coníferas en México. INIFAP-CIRNOC-Campo Experimental Pabellón. Pabellón de Artega, Ags., México. Folleto Técnico Núm. 74. 38 p.

Sánchez-Martínez, G. y J. F. Reséndiz-Martínez. 2020. Respuesta de Dendroctonus frontalis Zimmerman y Dendroctonus mexicanus Hopkins a dos atrayentes semioquímicos en la Sierra Gorda de Querétaro, México. Southwestern Entomologist 45(2):511-320. Doi: 10.3958/059.045.0219.

Sánchez S., J. A., L. M. Torres E., A. Cano P. y O. U. Martínez B. 2003. Daños y diversidad de insectos descortezadores de coníferas en el noreste de México. Ciencia Forestal en México 28(93): 41-56. https://cienciasforestales.inifap.gob.mx (12 de mayo de 2021).

Schowalter, T. D., E. Hansen, R. Molina and Y. Zhang. 1997. Integrating the ecological roles of phytophagous insects, plant pathogens and mycorrhizae in managed forests. In: Kohm, K. A. and J. F. Franklin (eds). Creating a forestry for the $21^{\text {st }}$ century. Island Press. Washington, D.C., USA. pp. 171-189.

Schowalter, T. D. 2012. Ecology and management of bark beetles (Coleoptera: Curculionidae: Scolytinae) in southern pine forests. Journal of Integrated Pest Management 3(2): 1-7. Doi:10.1603/IPM11025. 
Secretaria del Medio Ambiente y Recursos Naturales (Semarnat).2018. Norma Oficial Mexicana NOM-019-SEMARNAT-2017, que establece los lineamientos técnicos para la prevención, combate y control de insectos descortezadores. Diario Oficial de la Federación.

https://www.dof.gob.mx/nota_detalle.php?codigo=5516918\&fecha $=22 / 03 / 2018$ (15 de marzo de 2021).

Smith D. R. and M. R. Wagner. 1986. Recognition of two species in the pine feeding Neodiprion fulviceps Complex (Hymenoptera: Diprionidae) of Western United States. Proceedings of the Entomological Society Washington 88(2): 215-220.

Smith, D. R. 1988. A synopsis of the sawflies (Hymenoptera: Symphyta) of America south of the United States: Introduction Xyelidae, Pamphiliidae, Cimbicidae, Diprionidae, Xyphydriidae, Siricidae, Orussidae, Cephidae. Systematic Entomology 13: 205-261. Doi: 10.1111/j.1365-3113.1988.tb00242.x.

Smith, D. R., G. Sánchez M. and S. Ordaz S. 2010. A new Monoctenus (Hymenoptera: Diprionidae) damaging Juniperus flaccida (Cupressaceae) in San Luis Potosí, Mexico. Proceedings of the Entomological Society of Washington 112(3): 444-450. Doi: 10.4289/0013-8797.112.3.444.

Smith, D. R., G. Sánchez M. and A. Ojeda A. 2012. A new species of Zadiprion (Hymenoptera: Diprionidae) on Pinus durangensis from Chihuahua, Mexico, and a review of other species of the genus. Proceedings of the Entomological Society of Washington 114(2): 224-237. Doi: 10.4289/0013-8797.114.2.224.

Sutherland, J. R., T. Miller and R. Salinas Q. (eds.). 1987. Cone and seed diseases of North American conifers. North American Forestry Commission. Publication 1. Forestry Canada, Pacific and Yukon Region. Victoria, BC, Canada. 77 p. https://cfs.nrcan.gc.ca/publications?id=2708 (15 de marzo de 2021). 
Sturrock, R. N., S. J. Frankel, A. V. Brown, P. E. Hennon, J. T. Kliejunas, K. J. Lewis, J. J. Worrall and A. J. Woods. 2011. Climate change and forest diseases. Plant Pathology 60: 133-149. Doi: 10.1111/j.1365-3059.2010.02406.x.

Torres E., L. M., J. A. Sánchez S., A. Cano P. y O. U. Martínez B. 2004. Uso de feromonas en el manejo integrado del descortezador de pinos Dendroctonus adjunctus Blandford. INIFAP-CIRNE, Campo Experimental Saltillo. Saltillo, Coah., México. Folleto Técnico Núm. 13. 16 p.

Urcelay, C., G. Robledo, F. Heredia, G. Morera y F. García M. 2012. Hongos de la madera en el arbolado urbano de Córdoba. Instituto Multidisciplinario de Biología Vegetal (UNC-CONICET). Córdoba, Argentina. 104 p.

Vázquez C. I., G. Sánchez M. y S. Madrigal H. 2007. Fluctuación poblacional de Dendroctonus mexicanus Hopk. bajo dos condiciones de manejo forestal en Michoacán México. Ciencia Forestal en México 32(102): 57-78. http://cienciasforestales.inifap.gob.mx/editorial/index.php/forestales/article/view/7 50 (15 de marzo de 2021).

Velasco B., E., J. F. Reséndiz M., L. Sandoval C., L. P. Olvera C., F. Moreno S., M. V. Gutiérrez G. y O. Cedeño S. 2002. Diagnóstico sanitario de los bosques del Distrito Federal, México. Ciencia Forestal en México 27 (91): 7-26. http://cienciasforestales.inifap.gob.mx/editorial/index.php/forestales/article/view/906 (15 de marzo de 2021).

Velasco B., F., E. N. Cortés B., A. González H., F. Moreno S. y E. M. Benavides M. 2013. Diagnóstico y caracterización del arbolado del Bosque de San Juan de Aragón. Revista Mexicana de Ciencias Forestales 4 (19): 102-111.

Doi: $10.29298 /$ rmcf.v4i19.382. 
Villa C., J. 1985. Enemigos naturales y organismos asociados al descortezador de pinos Dendroctonus adjuntus blandford en el nevado de Colima. INIF. México, D.F., México. Boletín Técnico No. 121. 22p.

Villa C., J. 1992a. Atrayentes químicos en escarabajos descortezadores Dendroctonus mexicanus y D. adjunctus (Col.: Scolytidae). Ciencia Forestal en México 17 (71): 103-122.

http://cienciasforestales.inifap.gob.mx/editorial/index.php/forestales/article/view/1100 (15 de marzo de 2021).

Villa C., J. 1992b. Evaluación del control de Dendroctonus mexicanus mediante la fumigación con fosfamina. Ciencia Forestal en México. 17(72): 55-74. http://cienciasforestales.inifap.gob.mx/editorial/index.php/forestales/article/view/1065 (15 de marzo de 2021).

Villa C., J. 1993. Valoración del derribo y abandono como método de control para Dendroctonus adjunctus BIf- (Col.: Scolytidaet) en el Parque Nacional Nevado de Colima. SARH-INIFAP. México, D.F., México. Boletín Técnico Núm. 110. 71 p.

Vité, J. P., F. Islas S., J. A. A. Renwick, P. R. Hughes and R. A. Kliefoth. 1974. Biochemical and biological variation of southern pine beetle populations in North and Central America. Zeitschrift für Angewandte Entomologie 75: 422-435. Doi: 10.1111/j.14390418.1974.tb01863.x.

Williams, D. W. and A. M. Liebhold. 2002. Climate change and the outbreak range of two north American bark beetles. Agricultural and Forest Entomology 4: 87-99. https://www.nrs.fs.fed.us/pubs/jrnl/2002/ne_2002_williams-d_001.pdf (15 de marzo de 2021). 
Zhang, A., D. Amalin, S. Shirali, M. S. Serrano, R. A. Franqui, J. E. Oliver, J. A. Klun, J. R. Aldrich, D. E. Meyerdik and S. L. Lapointe. 2004. Sex pheromone of the Pink Hibiscus Mealybug, Maconelliccocus hirsutus, contains an unusual cyclobutanoid monoterpene. PNAS 101: 9601-9606. Doi: 10.1073/pnas.0401298101.

Zhang, A. and D. Amalin. 2005. Sex pheromone of the female Pink Hibiscus Mealybug, Maconellicoccus hirsutus (Green) (Homoptera: Pseudococcidae): Biological activity evaluation. Environmental Entomology 34: 264-270. Doi: 10.1603/0046-225X-34.2.264.

\section{(c) (1) (9)}

Todos los textos publicados por la Revista Mexicana de Ciencias Forestales -sin excepción- se distribuyen amparados bajo la licencia Creative Commons 4.0 Atribución-No Comercial (CC BY-NC 4.0 Internacional), que permite a terceros utilizar lo publicado siempre que mencionen la autoría del trabajo y a la primera publicación en esta revista. 